\title{
X.509 und der Gültigkeitszeitraum
}

Die Menschheit zerfällt in 10 Teile:

der erste drückt sich falsch aus,

und der zweite missversteht es.

Alexander Roda Roda

\section{Gültige Zertifikate}

Nach $\$ 2$ des Signaturgesetzes muss eine qualifizierte Signatur auf einem zum Zeitpunkt ihrer Erstellung gültigen qualifizierten Zertifikat beruhen.

Ein zu diesem Zeitpunkt gesperrtes Zertifikat ist sicher ungültig, aber ab wann und wie lange ist es eigentlich gültig?

Jedes zum Standard X.509/RFC 5280 konforme Zertifikat enthält immer zwei Datumsangaben, die als ASN.1-Variable notBefore und notAfter heißen und in einer validity genannten Folge zusammengefasst werden. In der deutschsprachigen Literatur wird diese Zeitspanne regelmäßig als Gültigkeitszeitraum übersetzt, obwohl sie formal nicht Beginn und Ende eines Zeitraums angibt, sondern nur einschränkt.

Ein Blick in den X.509, wie denn diese Zeitspanne, die Variable validity, tatsächlich definiert ist, lohnt sich:

- „Validity is the time interval during which the CA warrants that it will maintain information about the status of the certificate“.

Oder in freier Übersetzung:

> „Validity ist das Zeitintervall für das sich die CA verpflichtet, aktuelle Statusinformationen zum Zertifikat bereitzuhalten“.

Ein technischer Gültigkeitszeitraum wird dadurch sicher nicht beschrieben, sondern eher vielleicht eine Garantiedauer oder ein Gewährleistungszeitraum. Wir wollen ihn deshalb in diesem Beitrag auch so nennen.

Ein Zertifizierungsdiensteanbieter (ZDA/CA) legt folglich bei der Erstellung eines Zertifikats fest, ab wann er spätestens die Informationen zum Status des Zertifikats bereitstellen wird und wie lange er das mindestens zu tun beabsichtigt. Diese Informationen sind für eine Nutzerin auch wichtig, denn nur in diesem Zeitraum kann sie erwarten, dass ihr die Information, ob das Zertifikat überhaupt ausgestellt wurde und ob es, und falls wann, gesperrt wurde, bereitgestellt werden. Ohne diese Informationen kann sie dem Zertifikat eigentlich nicht vertrauen und prüfen, ob eine Signatur gültig ist.

\section{Vor notBefore}

Ab dem Zeitpunkt notBefore ist ein Zertifikat gültig, falls es nicht schon gesperrt ist. Ob es davor schon gültig war, kann man selten direkt nachprüfen, da es dazu keine gesicherten Informationen geben muss. Es geht manchmal nur über einen Umweg, wie bei den Zertifikaten $0 \times 313,0 \times 314$ und $0 \times 315$ der Bundesnetzagentur, denen jeweils die folgenden notBefore-Daten zugeordnet sind:

\begin{tabular}{|l|l|l|l|}
\hline $\mathbf{N r}$ & notBefore & Subj & Issu \\
\hline $0 \times 313$ & $2007-05-25$ 11:01:44 & $12 R$ & $12 R$ \\
\hline $0 \times 314$ & $2007-05-2907: 57: 50$ & $11 R$ & $12 R$ \\
\hline $0 \times 315$ & $2007-05-2909: 17: 45$ & $12 R$ & $11 R$ \\
\hline
\end{tabular}

Signiert wurden jeweils die Schlüssel von und durch 11R-CA und 12R-CA. Das Zertifikat $0 \times 313$ ist also selbst-, $0 \times 314$ ist alt-von-neu- und $0 \times 315$ ist neu-von-altsigniert. Wenn jetzt das letzte Zertifikat nicht schon vor seinem notBefore Zeitpunkt gültig gewesen wäre, wären die Signaturen von $0 \times 313$ und $0 \times 314$ nicht qualifiziert, weil der Schlüssel 12R-CA bei seiner Verwendung nicht auf einem qualifizierten Zertifikat beruht hätte.

Aus der technischen Sicht des X.509 ist eine Antwort auf die Frage, ab wann ein Zertifikat zum ersten Mal gültig ist, ohne Bedeutung, entscheidend ist die Prüfbarkeit der darauf beruhenden Signaturen, und die ist verlässlich erst innerhalb des Gewährleistungszeitraums gegeben.

\section{Im Gewährleistungszeitraum}

Die Prüfung einer Signatur enthält als wesentlichen Bestandteil die Prüfung der Zertifikatskette zum Vertrauensanker, die manchmal auch als Gültigkeitsprüfung des Zertifikats bezeichnet wird. Tatsächlich wird dabei das Vertrauensniveau für das Zertifikat des Signierenden bestimmt. Eine Zertifikatskette ist „gültig“, wenn das erforderliche Niveau erreicht wurde. Im Unterschied zu einer Gültigkeitsprüfung einer Signatur ist, wenn über die angegebene Kette das entsprechende Vertrauensniveau nicht erreicht wird, das geprüfte Zertifikat aber nicht gleich ungültig, wenn bestimmte Prüfinformationen nicht verfügbar, veraltet oder verblasst sind oder die ganze Kette ungeeignet ist, weil sie nicht in einem Vertrauensanker endet.

Da aber für den gesamten Gewährleistungszeitraum vom ZDA zugesichert wird, dass der Vertrauensstatus des Zertifikats zweifelsfrei feststellbar ist, könnte man ihn noch Vertrauensstatusbestimmbarkeitszeitraum nennen oder, da ja die Gültigkeit einer Signatur auf dem Vertrauensstatus des Signaturzertifikats beruht, vielleicht auch Mindestsignaturgültigkeitsprüfzeitraum?

\section{Nach notAfter}

Nach notAfter müssten Statusinformationen nicht mehr durch den ZDA zur Verfügung gestellt werden. Aber das Signaturgesetz fordert die Verfügbarkeit dieser Informationen über den Gewährleistungszeitraum hinaus, also seine Verlängerung, denn abgelaufene oder auch gesperrte Zertifikate sollen weiter zur Gültigkeitsprüfung von Signaturen und zur Bestimmung des Vertrauensstatus herangezogen werden können.

Das Signaturgesetz verwendet den Begriff des gültigen Zertifikats außer in $\$ 2$ nur noch an einer Stelle, aber da ungenau, denn es wird in $\$ 13$ bei Einstellung der Tätigkeit nur gefordert, dass die gültigen Zertifikate von einem anderen ZDA zu übernehmen sind. Tatsächlich müssen aber alle, auch die gesperten Zertifikate übernommen werden, zumindest die, deren Gewährleistungszeitraum noch nicht abgelaufen ist.

Man könnte Missverständnisse zukünftig dadurch vermeiden, indem man grundsätzlich auf den Begriff des gültigen Zertifikats verzichtet, wie etwa so:

-... Signatur, die auf einem qualifizierten Zertifikat beruht, welches zum Zeitpunkt ihrer Erzeugung nicht gesperrt und dessen Gewährleistungszeitraum zugleich auch noch nicht abgelaufen war.

Obwohl es sich eingebürgert hat, dass man ein Zertifikat nach not After oder nach der Sperrung als ungültig bezeichnet, sollte man nie vergessen, dass aus technischer Sicht ein Zertifikat nur einen Gewährleistungs- und keinen Gültigkeitszeitraum enthält. 